\title{
Sarcoidosis of the Ear, Nose and Throat: a review of the literature
}

\author{
Nicholas Cereceda-Monteoliva ${ }^{1}$, Maral Rouhani ${ }^{2}$, Elizabeth Maughan ${ }^{2}$, Anthony Rotman ${ }^{2}$, \\ Nara Orban ${ }^{3}$, Chadwan Al Yaghchi ${ }^{1}$, and Guri Sandhu ${ }^{1}$ \\ ${ }^{1}$ Imperial College Healthcare NHS Trust \\ ${ }^{2}$ Charing Cross Hospital \\ ${ }^{3}$ Barts Health NHS Trust
}

January 4, 2021

\begin{abstract}
Objectives. Sarcoidosis is a multisystemic inflammatory disease with extrathoracic manifestations, most commonly affecting the young and middle-aged, female and black populations. Diagnosis usually requires evidence of non-caseating granulomata and, when treated, prognosis is usually favourable. We aim to establish the incidence, clinical features and optimal treatment of ENT manifestations of this disease. Design. We performed a review of the literature to determine the evidence-base supporting this. Results. ENT manifestations are present in 10-15\% of patients with sarcoidosis, often as a presenting feature, and require vigilance for swift recognition and coordinated additional treatment specific to the organ. Laryngeal sarcoidosis presents with difficulty in breathing, dysphonia and cough, and may be treated by Speech and Language Therapy (SLT) or intralesional injection, dilatation or tissue reduction. Nasal disease presents with crusting, rhinitis, nasal obstruction and anosmia, usually without sinus involvement. It is treated by topical nasal or intralesional treatments but may also require endoscopic sinus surgery, laser treatment or even nasal reconstruction. Otological disease is uncommon but includes audiovestibular symptoms, both sensorineural and conductive hearing loss, and skin lesions. Conclusions. The consequences of ENT manifestations of sarcoidosis can be uncomfortable, disabling and even life threatening. Effective management strategies require good diagnostic skills and use of specific therapies combined with established treatments such as corticosteroids. Comparisons of treatment outcomes are needed to establish best practice in this area.
\end{abstract}

\section{Abstract \\ Objectives .}

Sarcoidosis is a multisystemic inflammatory disease with extrathoracic manifestations, most commonly affecting the young and middle-aged, female and black populations. Diagnosis usually requires evidence of non-caseating granulomata and, when treated, prognosis is usually favourable. We aim to establish the incidence, clinical features and optimal treatment of ENT manifestations of this disease.

\section{Design .}

We performed a review of the literature to determine the evidence-base supporting this.

\section{Results.}

ENT manifestations are present in 10-15\% of patients with sarcoidosis, often as a presenting feature, and require vigilance for swift recognition and coordinated additional treatment specific to the organ. Laryngeal sarcoidosis presents with difficulty in breathing, dysphonia and cough, and may be treated by Speech and Language Therapy (SLT) or intralesional injection, dilatation or tissue reduction. Nasal disease presents with crusting, rhinitis, nasal obstruction and anosmia, usually without sinus involvement. It is treated by topical nasal or intralesional treatments but may also require endoscopic sinus surgery, laser treatment or 
even nasal reconstruction. Otological disease is uncommon but includes audiovestibular symptoms, both sensorineural and conductive hearing loss, and skin lesions.

\section{Conclusions .}

The consequences of ENT manifestations of sarcoidosis can be uncomfortable, disabling and even life threatening. Effective management strategies require good diagnostic skills and use of specific therapies combined with established treatments such as corticosteroids. Comparisons of treatment outcomes are needed to establish best practice in this area.

\section{Keywords}

Sarcoidosis, Otorhinolaryngologic Diseases, Larynx, Granuloma

\section{Objectives}

Sarcoidosis is a chronic inflammatory disease of unknown aetiology, characterised by the formation of noncaseating granulomata involving one or more organs. Extrathoracic manifestations of the disease can include otorhinolaryngological problems, with a particular predilection for upper and lower airway involvement. This article will outline the background of sarcoidosis, describe recognised ENT manifestations and discuss current treatment options.

\section{Design}

We performed a review of the literature to determine the evidence-base surrounding the management of ENT manifestations of this disease. We report the background of sarcoidosis first followed by the implications in ENT.

\section{Results - Sarcoidosis \\ Epidemiology}

The prevalence of sarcoidosis varies geographically between 1-40 cases per 100,000 and is more prevalent in south-eastern USA and Scandinavia. It occurs more commonly in a bimodal distribution affecting the young and middle-aged populations, females are affected more frequently than men, and black people more than white (1).

\section{Pathophysiology}

The cause of sarcoidosis is not yet well understood but an antigen-triggered, cell-mediated immune response is known to initiate the sarcoidosis disease process (2). Genetic susceptibility and specific environmental or infectious triggers combine to perpetuate a chronic cell-mediated immunological response (3). T lymphocytes and macrophages accumulate and produce inflammatory mediators such as cytokines (particularly TNFalpha, IL-12 and IL-18), which lead to the formation of granulomata $(2,4)$. Certain HLA haplotypes are suggested to predispose to the disease and bacterial triggers have also been proposed, although evidence for this remains inconclusive $(1,2,5)$.

\section{Diagnosis}

Sarcoidosis is diagnosed on the basis of clinical and radiological suspicion, combined with biopsy evidence of non-caseating granulomata, in the absence of any other cause of granulomatous disorders (1).

The Kveim reaction is important as a clinical observation in making a diagnosis of sarcoidosis $(3,4)$, however its use has fallen out of favour over the years. Imaging techniques routinely employed include CT (HRCT is particularly useful in chest sarcoid diagnosis) and MRI (with gadolinium contrast) $(6,7)$. Biopsy typically yields multiple epithelioid cell granulomata made from mononuclear cells, with variable degrees of necrosis, leucocyte infiltration and hyaline fibrosis (4). Supporting evidence can include a high CD4/CD8 T lymphocyte ratio on bronchoalveolar lavage $(1,2)$. 
Blood tests can provide supporting evidence for the diagnosis of sarcoidosis. Serum Angiotensin-converting enzyme (ACE) levels are often raised in sarcoidosis, however the nonspecific and insensitive nature of this rise relegates its use to monitoring the course of the disease (8). Hypercalcaemia may also be seen (1) and other acute phase reactants, such as ESR, may also be raised.

\section{General Clinical Manifestations}

Intrathoracic manifestations of sarcoidosis include pulmonary infiltrates and hilar lymphadenopathy, as well as cardiac sarcoidosis (more common in older Japanese females), which can be life-threatening $(1,7)$. Extrathoracic disease occurs in around $50 \%$ of patients, and virtually every organ can be affected (1). The extent and degree of disease is extremely variable between patients. Skin, lymph node, eye and liver involvement are most common outside of the thorax, each being found in between 10-25\% of patients (1).

Around $10-15 \%$ of patients with sarcoid have ENT manifestations of sarcoidosis $(1,5,9)$ which may be the presenting symptom of their disease (10). The differential often includes vasculitides such as granulomatosis with polyangiitis, formerly called Wegener's, or eosinophilic granulomatosis with polyangiitis, formerly Churg-Strauss syndrome, granulomas of infective origin (such as tuberculosis, aspergillosis, or actinomycosis), and inflammatory diseases with extrasystemic manifestations, such as Crohn's disease, which should be excluded (4). Sinonasal sarcoidosis is rare (occurring in 1-4\% of patients), but has been described in numerous case studies, and is a well-recognised chronic and stubborn form of the disease $(8,10,11)$. Sarcoidosis can also involve the larynx, salivary glands and ear in rare cases $(3,12-16)$.

\section{Treatment principles}

Medical management with oral corticosteroids is the mainstay of treatment in generalised sarcoidosis and in severe otolaryngologic disease $(1,9,17)$. However, the risks of uncontrolled disease need to be balanced against the incumbent risks and side-effects of long-term corticosteroid therapy (10). Adjunctive or alternate drug therapies have therefore also been used in order to reduce steroid doses $(18,19)$. These include:

- Cytotoxic agents (such as methotrexate, azathioprine, cyclophosphamide and chlorambucil)

- Anti-malarial drugs (chloroquine and hydroxychloroquine)

- TNF-alpha inhibitors (infliximab, adalimumab, etanercept).

No control studies exist as yet comparing these therapies to steroid treatment, but effective treatment is reported anecdotally in cases of sarcoidosis with extrathoracic manifestations such as lupus pernio and uveitis $(18,19)$. Furthermore, specific treatments can be helpful in the local management of otorhinolaryngologic sarcoidosis, and these are discussed below.

\section{Results - ENT manifestations of disease}

\section{Laryngeal Sarcoidosis}

Laryngeal sarcoidosis is particularly rare, with a large cohort of patients with systemic sarcoidosis demonstrating an incidence of only $0.6 \%$ (14). As with other manifestations of sarcoidosis, age at presentation is typically from 20 - 40 years old (13).

The presenting complaint in the majority of patients relates to difficulty breathing and less commonly, dysphonia and cough. Pain is not usually a feature. Only a small proportion of patients complain of dysphagia and if so, it is rarely the sole symptom. Those with dysphagia inevitably modify their diet and some silently aspirate. Given the natural history and slow progression of this granulomatous disease, emergency department presentations are rare. Case reports of tracheostomy exist $(20,21)$, however most patients present with a gradual decline. Stridor is common but mild. Although symptom onset is gradual, the impact on quality of life is significant with a reduction in exercise capacity, compounding respiratory disease in affected young patients.

Endoscopic examination typically reveals supraglottic swelling and deformity, with the epiglottis, arytenoids and aryepiglottic folds involved, as demonstrated in Figure 1. A 'turban'-shaped epiglottis is typically 
described (21), due to the resultant morphological change after inflammatory and granulomatous infiltrate. True vocal fold paresis is rare but there can often be an appearance of restricted glottic mobility contributing to dysphonia due to the inflammatory process and resultant thickening.

Prior to a diagnosis of laryngeal sarcoidosis, a broad differential must be considered given that presenting symptoms related to airway and voice are also seen in many more common laryngeal pathologies including vocal fold paralysis, supraglottitis and laryngotracheal stenosis $(15,21)$. The laryngeal appearance may resemble acute infection or other granulomatous diseases such as tuberculosis and vasculitis. Malignancy may rarely present in this way and must always be considered. It is crucial that the entire respiratory tract has been assessed, as more foci of disease suggest a systemic process such as sarcoidosis, where solitary lesions are more likely to be due to localised pathology. Investigations may be performed but only a biopsy demonstrating non-caseating granulomata is diagnostic (5).

Conservative options typically relate to speech and language therapy for patients with symptomatic dysphonia or dysphagia (15). Medical therapy for those with pre-existing sarcoidosis has often been initiated as described above. The majority of patients that require surgical intervention have problematic airway symptoms. Microlaryngoscopy and biopsy is performed for a definitive diagnosis, whilst also providing an opportunity to dilate the airway if required. There have been descriptions of intralesional steroid injection, as well as the use of mitomycin-c to prevent recurrent scar (21). In general, surgical techniques employ the $\mathrm{CO} 2$ laser for tissue reduction or excision - the addition of the previously described 'pepper pot' technique has been demonstrated to reduce patients' Medical Research Council dyspnoea grade (21).

\section{Sinonasal Sarcoidosis}

Sinonasal involvement occurs in $1-4 \%$ of sarcoidosis cases $(8,11)$. Involvement tends to be mucosal but can involve the bone of the nose and paranasal sinuses. Typical symptoms include chronic crusting rhinitis (70-90\%), nasal obstruction (unilateral or bilateral) (80-90\%), and anosmia (70\%). Epistaxis (2\%) and nasal deformity can occur in advanced and destructive cases (22).

Clinical examination may discover hypertophy and a characteristic purplish colouring of the nasal mucosa, with granulations on the septum and inferior turbinates. Less commonly, the paranasal sinuses may be partially or completely opacified, with mucosal thickening and osteomeatal obstruction $(8,11)$. There have also been case reports of sinonasal sarcoidosis with peripheral nerve involvement, salivary gland extension and intracranial extension $(3,5)$. The sinonasal cavity are commonly affected in vasculitic conditions and these are therefore the primary differential diagnosis, in addition to chronic rhinosinusitis, prior to a tissue diagnosis.

Treatment of sinonasal sarcoidosis depends on the location and severity of the disease. Hence, a staging system is described by Krepsi et al. as shown in Table 1 (44). Medical nasal, intralesional and systemic treatments have been tried separately and in combination, with topical nasal and intralesional corticosteroid therapy having the benefit of avoiding the complications that occur with the use of systemic corticosteroids $(8,17)$. However, if the symptoms and clinical destruction are severe, systemic corticosteroids, as outlined above, are indicated.

Surgical treatment can be effective where medical treatment has failed, and in particular in cases which develop anatomical blockage of sinus drainage pathways with sarcoidosis lesions. Endoscopic sinus surgery can markedly improve quality of life and reduce the need for systemic steroids, but it will not eradicate the disease or prevent recurrence. Successful laser surgery with a CO2 laser has been reported, as well as successful partial nasal reconstruction with a two-stage, right-sided paramedical forehead flap with a rib cartilage framework $(8,10,11,18,19,22)$.

\section{Otologic Sarcoidosis}

Audiovestibular symptoms of sarcoidosis are uncommon but can include sensorineural hearing loss, vertigo, gait disturbance and disequilibrium from neurosarcoidosis affecting the vestibulocochlear nerves (16). Middle ear sarcoidosis has also been reported as a sentinel manifestation of the disease (23), presenting with tinnitus, 
hearing loss, purulent otorrhoea and otalgia. In this scenario, audiometric findings are mixed or conductive in nature, with CT demonstrating soft tissue masses in the tympanic cavity extending into the mastoid antrum and air cells. While skin is commonly involved in sarcoidosis, the external ear is seldom involved $(12,24)$. Cases described include tender and non-tender raised nodules, swelling or inflammation that can affect the helix, lobules or external auditory canal $(12,24)$.

Otologic symptoms usually improve with oral or intralesional corticosteroid therapy (12). Infliximab, a TNF- $\alpha$ blocking agent, has also been reported as successful in treating ear lobe sarcoidosis refractory to nonbiologic immunosuppressants and intralesional injected corticosteroids (24). Case reports have suggested that surgical excision of granulation tissue alongside systemic steroid therapy can result in improvement of symptoms (23).

\section{Prognosis}

Sarcoidosis generally shows spontaneous remission in $12-36$ months (50-60\% of cases). Prognosis is usually favourable, although a $3-9 \%$ mortality is reported, usually from lung or cardiac complications $(7,17)$. It is difficult to recommend ENT-specific treatments given the scarcity of data that exists on the management and prognosis of site-specific manifestations. For example, laryngeal sarcoidosis is a rare head and neck manifestation of what is already a rare condition. Since most patients with sarcoidosis do not have ENT manifestations of their disease, there is little data regarding rates of remission. As such, there are only limited case series with which to base best practice.

\section{Conclusions}

The consequences of ENT manifestations of sarcoidosis can be uncomfortable, disabling and disfiguring and those affecting the larynx can be life threatening $(10,20)$. The coordinated management of these requires good diagnostic skills and multimodal treatment. Established treatments using corticosteroids are observed to be effective but not without risks of long-term use. Intralesional treatments are commonly reported and surgical excision of tissue may be necessary.

For clinical care to evolve and improve, it is vital for affected patients to be managed using all the resources available to the multidisciplinary team and that outcomes are studied and recorded so as to establish best practice in this area.

\section{Acknowledgements}

The authors would like to thank colleagues who shared their clinical experience and encouraged this research.

\section{Disclosure Statement}

The authors have no potential sources of conflict of interest to declare.

\section{References}

1. Nunes H, Bouvry D, Soler P, Valeyre D. Sarcoidosis. Orphanet J Rare Dis. 2007;2:46.

2. Chen ES, Moller DR. Etiology of sarcoidosis. Clin Chest Med. 2008;29(3):365-77, vii.

3. Mrówka-Kata K, Kata D, Lange D, Namysłowski G, Czecior E, Banert K. Sarcoidosis and its otolaryngological implications. European Archives of Oto-Rhino-Laryngology. 2010;267(10):1507-14.

4. Rosen, Y. The Pathology of Sarcoidosis. Seminars in respiratory and critical care medicine. 2007;28:36-52.

5. Badhey AK, Kadakia S, Carrau RL, Iacob C, Khorsandi A. Sarcoidosis of the head and neck. Head Neck Pathol. 2015;9(2):260-8.

6. van Beek EJ, Hoffman EA. Functional imaging: CT and MRI. Clinics in chest medicine. 2008;29(1):195216. 
7. Kirkil G, Lower EE, Baughman RP. Predictors of Mortality in Pulmonary Sarcoidosis. CHEST. 2018;153(1):105-13.

8. Braun JJ, Gentine A, Pauli G. Sinonasal sarcoidosis: review and report of fifteen cases. The Laryngoscope. 2004;114(11):1960-3.

9. Aladesanmi OA. Sarcoidosis: an update for the primary care physician. MedGenMed. 2004;6(1):7.

10. Gulati S, Krossnes B, Olofsson J, Danielsen A. Sinonasal involvement in sarcoidosis: a report of seven cases and review of literature. European Archives of Oto-Rhino-Laryngology. 2012;269(3):891-6.

11. Aubart FC, Ouayoun M, Brauner M, Attali P, Kambouchner M, Valeyre D, et al. Sinonasal involvement in sarcoidosis: a case-control study of 20 patients. Medicine. 2006;85(6):365-71.

12. Adelola OA, Fernandez R, Ahmad R, Leamy M, Moriarty BG. Sarcoidosis of the external ear-literature review and report of a case. J Laryngol Otol. 2007;121(3):289-92.

13. Duchemann B, Lavolé A, Naccache J-M, Nunes H, Benzakin S, Lefevre M, et al. Laryngeal sarcoidosis: a case-control study. Sarcoidosis, vasculitis, and diffuse lung diseases: official journal of WASOG. 2014;31(3):227.

14. Neel III HB, McDonald TJ. Laryngeal sarcoidosis: report of 13 patients. Annals of Otology, Rhinology \& Laryngology. 1982;91(4):359-62.

15. Mayerhoff RM, Pitman MJ. Atypical and disparate presentations of laryngeal sarcoidosis. Annals of Otology, Rhinology \& Laryngology. 2010;119(10):667-71.

16. Colvin I. Audiovestibular manifestations of sarcoidosis: a review of the literature. The Laryngoscope. $2006 ; 116(1): 75-82$.

17. Baughman RP. Pulmonary sarcoidosis. Clinics in chest medicine. 2004;25(3):521-30, vi.

18. Callejas-Rubio JL, López-Pérez L, Ortego-Centeno N. Tumor necrosis factor-alpha inhibitor treatment for sarcoidosis. Therapeutics and Clinical Risk Management. 2008;4(6):1305.

19. Roberts SD, Wilkes DS, Burgett RA, Knox KS. Refractory sarcoidosis responding to infliximab. Chest. 2003;124(5):2028-31.

20. Ryu C, Herzog EL, Pan H, Homer R, Gulati M. Upper Airway Obstruction Requiring Emergent Tracheostomy Secondary to Laryngeal Sarcoidosis: A Case Report. Am J Case Rep. 2017;18:157-9.

21. Butler CR, Nouraei SR, Mace AD, Khalil S, Sandhu SK, Sandhu GS. Endoscopic airway management of laryngeal sarcoidosis. Archives of Otolaryngology-Head \& Neck Surgery. 2010;136(3):251-5.

22. Gurkov R, Berghaus A. Nasal reconstruction in advanced sinunasal sarcoidosis. Rhinology. 2009;47(3):327.

23. Arsovic N, Babic B, Dimitrijevic M, Bukurov B, Vucinic V. Otitis media and facial paralysis as presenting symptoms of a primary middle ear sarcoidosis. Otol Neurotol. 2013;34(8):e121-2.

24. Vorselaars A, Keijsers R, Grutters J. Earlobe sarcoidosis. Sarcoidosis Vasculitis and Diffuse Lung Diseases. $2012 ; 29(1): 55$.

25. Krespi YP, Kuriloff DB, Aner M. Sarcoidosis of the Sinonasal Tract: A New Staging System. Otolaryngology-Head and Neck Surgery. 1995;112(2):221-7.

\section{Tables}

Table 1. Staging of sinonasal sarcoidosis, as proposed by Krepsi et al. (25):

Stage 1 Mild, reversible disease without paranasal sinus involvement

Stage 2 Moderate, potentially reversible disease without sinus involvement 


\section{Figure Legends}

Figure 1. Endoscopic appearance of laryngeal sarcoidosis with pale oedematous supraglottic swelling and deformity affecting the epiglottis with arytenoids and aryepiglottic folds involved to a lesser extent.

\section{Key Points}

- Sarcoidosis is a multisystemic inflammatory disease with ENT manifestations present in $10-15 \%$ of patients, often as a presenting feature.

- Diagnosis typically requires evidence of non-caseating granulomata and prognosis is favourable with treatment.

- 'Turban-shaped' epiglottis and restricted glottic mobility is observed on endoscopic examination of laryngeal sarcoidosis, where treatments include Speech and Language Therapy (SLT), intralesional injection, dilatation and tissue reduction surgery.

- Sinonasal sarcoidosis is staged according to location and severity of disease, which guides treatment options, including intranasal, intralesional and systemic treatments, separately or in combination with topical, nasal and intralesional corticosteroid, and endoscopic sinus surgery.

- Otologic sarcoidosis is difficult to diagnose but responds well to oral or intralesional corticosteroid therapy.

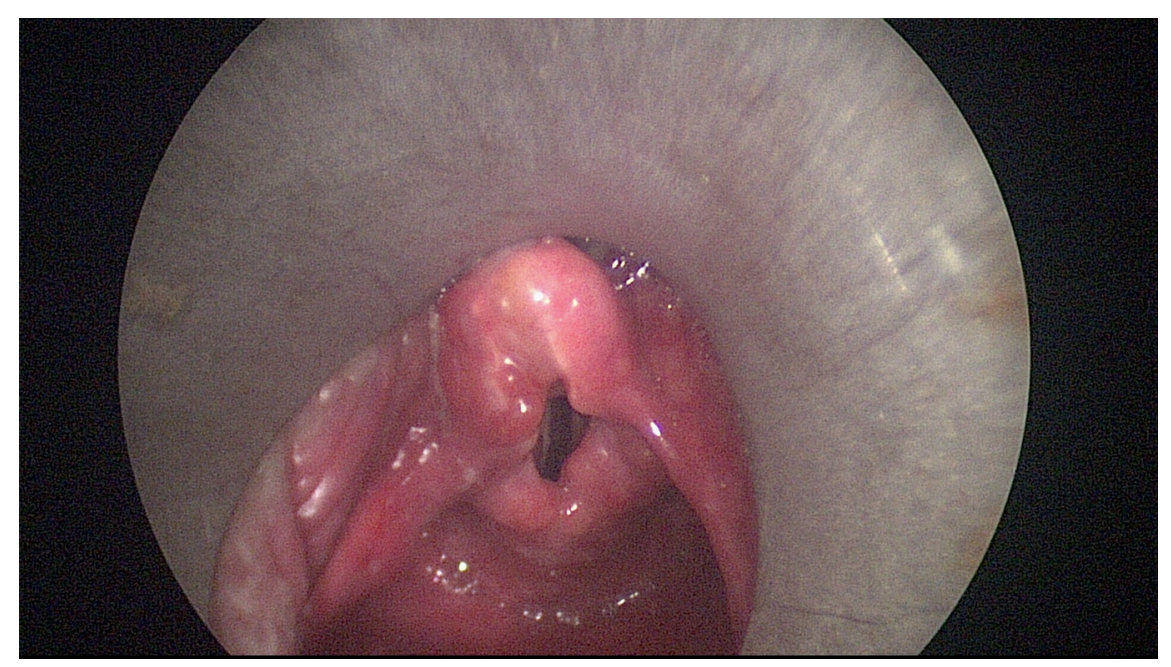

